\title{
Nonlinear analysis of high accuracy and reliability in traffic flow prediction
}

https://doi.org/10.1515/nleng-2020-0016

Received Feb 2, 2019; accepted Apr 2, 2020.

\begin{abstract}
For quantitatively identifying the chaotic patterns in traffic flow prediction, certain types of Duffing systems can be used. The accuracy and reliability of numerical results of the system's solution have significant influence on the traffic flow prediction. The nonlinear dynamic behavior of Duffing system used for the traffic flow prediction is investigated in this research. The solutions of the system are developed and solved numerically by using the P-T method. The regular and irregular responses of the system considered are graphically illustrated with the newly developed P-R method. Based on the results of the research, the frequency and amplitude of the external excitations applied on the system significantly affecting the nonlinear dynamic behavior therefore the traffic flow prediction in transferring the results by WignerVille transform. Additionally, a comparison between the P$\mathrm{T}$ and Runge-Kutta method is conducted in regarding the accuracy and reliability of the methods.
\end{abstract}

Keywords: traffic flow prediction, nonlinear dynamic system, accuracy and reliability

\section{Introduction}

As the fast development of vehicle manufacturing and road construction, the numbers of vehicles owned and used by people soars in most of the cities. As a result, keeping the traffic flow fluent has turned into a key role in modern society where the busy traffic can be pain and gives negative effect on both engineering and social activities. With that being side, timely traffic flow information with high accuracy was in extensive need, espe-

\footnotetext{
*Corresponding Author: Liming Dai, Industrial Systems Engineering, University of Regina, Regina, SK S4S 0A2, Canada, E-mail: liming.dai@uregina.ca, Tel: 306.585-4498, Fax: 306.585-4855 Luyao Wang, College of Computer, Qinghai Normal University, Xining, Qinghai 810008, China Industrial Systems Engineering, University of Regina, Regina, SK S4S 0A2, Canada
}

cially for individual travelers, business sectors and government agencies [1]. Traffic flow prediction, which collects the traffic data and use it to predict what the future traffic patterns could be, has increasingly attracted attention from researchers and engineers. The accuracy and reliability of the traffic flow prediction is significant, especially when the rapid development of Intelligence Transportation Systems (ITS) is considered. The prediction was considered as "a critical part for the successful deployment of ITS subsystems, particularly advanced traveler information systems, advanced traffic management systems, advanced public transportation systems, and commercial vehicle operations" [2].

However, as a random system in general, many factors affect traffic flow. The traffic flow therefore involves uncertainty and complexity [3]. Currently, there exist many traffic flow prediction systems and models conducted by peer researchers and engineers. For advanced traffic management system and advanced traveler information system, Yang et al. [4] conducted an investigation for shorttime traffic flow prediction. Their method achieved a better performance in multistep prediction over the conventional methods. A short term prediction of traffic flow was conducted with applications of Artificial Neural Network (ANN) and the past traffic data. Wigner-Ville distribution was applied recently by Mrgole \& Drago [3] for identifying chaotic patterns in traffic flow prediction, where a Duffing system was used. By solving the Duffing's system and transferring the results by Wigner-Ville transform, the reliable chaotic pattern in the traffic flow can be successfully recognized. Therefore, the accuracy of the numerical solution of the Duffing's system played a significantly influential role in the overall traffic flow prediction. It is noticed that Runge-Kutta method was utilized in Mrgole \& Drago's work for solving the Duffing's system. However, accuracy and reliability of the Runge-Kutta method may raise some concerns especially when nonlinear and complex systems are considered.

The research about the accuracy of the Runge-Kutta method can be found as early as 1971, such as the studies made by Shampine and Watts [5], in which an innovative approach was presented for comparing local error estimators with consideration of the $4^{\text {th }}$ order Runge-Kutta 
method. In Hull et al. [6], the accuracy of the Runge-Kutta method in solving first order ordinary differential equations was investigated through a comparison with different numerical methods. With the test for accuracy and reliability of conventional integrations, the Runge-Kutta method was recognized with some shortcomings. Also, it was found that the $4^{\text {th }}$ order even $5^{\text {th }}$ order RungeKutta methods were suitable for merely solving certain types of problems, which focused on easy functions and required loose accuracy. In fact, Li and Darby [7] found that Runge-Kutta method may cause calculation errors and even unavoidable inaccuracy. Moreover, it should be noted that Runge-Kutta method generates results which are actually discrete. Several continuous approaches utilizing the Runge-Kutta method were indeed seen in the field, e.g., Enright and Hayes [8]. Nevertheless, their results can only be approximate. In Dai and Wang [9], additionally, RungeKutta method was found may lead to less accurate and sometimes unreliable even incorrect results in solving for nonlinear behavior of dynamic systems.

To overcome the shortages of these numerical methods, a newly developed method called price-wise constant (P-T) method was introduced by Dai [10]. It can not only provide continuous results, but also keep the results with high accuracy and reliability [9]. Together with PeriodicityRatio (PR) method, which is applied to recognize the characteristics of different motions, they can be a powerful tool set in analyzing the behaviors of nonlinear dynamic systems in a large number of fields. In Dai and Zhang [11], with the employment of P-T and PR method, an articulated pipe jointed with oil conveying system was studied and its nonlinear behaviors were analyzed with details under different conditions. By development of a secondary Poincare map approach combining the PR method, Huang et al. [12] systematically and completely identified the characteristics of an ecological oscillatory system with consideration of large ranges of system parameters. Recently, Dai and Xia [13] further developed the PR method by integration with Lyapunov exponent method. This novel approach was proved to provide higher completeness, efficiency and accuracy in diagnosing the nonlinear dynamic systems.

The present research focuses on the application of Duffing's system in traffic flow prediction. The P-T method is to be utilized for numerically solving and analyzing the system. The accuracy and reliability of the numerical solutions obtained, for the nonlinear dynamic systems subjected to external excitations, are to be investigated with the comparison between Runge-Kutta and the P-T method. Furthermore, the influence of the system parameters on the accuracy of the numerical calculations is to be studied. For identifying and analyzing the nonlinear behavior of the system, the P-R method is to be utilized in the research, for graphically illustrating regular and irregular responses of the system corresponding to various system parameters. The approach of the present research and the results generated provide significant guidance for conducting traffic flow prediction with high accuracy and reliability.

\section{Governing equation and numerical solutions}

In order to identify and analyze the chaotic patterns in traffic flow prediction practice, Wigner-Ville distribution is a consideration as described previously. Based on its unique characteristics, employment of Duffing's equation is natural selection. Researchers, such as Mrgole \& Drago [3], use a Duffing's system mathematically expressed in the following form for the investigations in identifying and analyzing the nonlinear patterns in traffic flow prediction.

$$
\ddot{x}+k \dot{x}-x^{3}+x^{5}=r \cos (\omega t)
$$

In the equation, $k$ is the damping ratio, $r$ and $\omega$ represents respectively the amplitude and frequency of the external excitation applied on the system. The external excitation expressed by the term $r \cos (\omega t)$ provides energy to the system and dominantly affect its behavior. For such a system, as noted by Kovacic and Brennan [14], the range of the damping ratio $k$ falls in between 0.2 and 0.5 . The purposes of this research are to identify the dynamic behaviors, especially chaotic pattern in traffic flow system under different external excitation, angular velocity and external excitations, through the approaches of the P-T and P-R methods.

The semi-analytical and numerical approach known as the P-T method is applied in the research to derive the numerical solutions of the nonlinear dynamic system presented in Eq. (1). As a numerical method with high accuracy, the P-T method was first introduced by Dai and Singh [15] to bring some advantages in solving such nonlinear dynamic systems. In using the P-T method, the original continuous governing equation is divided into a number of segments by introducing a piecewise constant argument $[N t] / N$ and linearized on each time interval, $[N t] / N \leq$ $t<([N t]+1) / N$, in which a linear dynamic system is developed. In the above expressions, $N$ can be considered as a positive integer that controls the time interval and thus determines the computational accuracy of the results. Theoretically, following relationship is evident and proved true by Dai and Singh [15]. It implies that a piecewise linear system can be related to its corresponding continuous 
systems by the following relationship as a bridge.

$$
\lim _{N \rightarrow \infty} \frac{[N t]}{N}=t
$$

A natural conclusion can therefore be given as that the numerical solution provided by the P-T method can reach the desired accuracy so long as $N$ is large enough. Moreover, the solutions generated by the P-T method are expected to be the exact solution as $N$ approaches infinity.

The concept of the P-T method is to linearize as less as possible the nonlinear dynamic system, for the purpose of maintaining as more as possible the original physical information of the nonlinear system. As such, the numerical results obtained can be more accurate and reliable in comparing with that of the other numerical methods existing in the market.

Specifically, the governing equation Eq. (1), per the P-T method, can be rewritten by

$$
\ddot{x}_{i}(t)+k \dot{x}_{i}(t)=f(x, \dot{x}, t)
$$

in the $i$ th time interval, in which

$$
f(x, \dot{x}, t)=x_{i}^{3}-x_{i}^{5}+r \cos (\omega t)
$$

To create the recurrence relations for numerical calculations, the local initial conditions are given in the following form.

$$
x_{i}\left(\frac{i}{N}\right) \triangleq d_{i}, \quad \dot{x}_{i}\left(\frac{i}{N}\right) \triangleq v_{i}
$$

Furthermore, the Taylor expansion can be applied as per the P-T method, such that the linearized governing equation Eq. (3), can be given by

$$
\begin{aligned}
& \ddot{x}_{i}(t)+k \dot{x}_{i}(t)=f_{[N t] / N}+f_{[N t] / N}^{\prime}\left(t-\frac{[N t]}{N}\right) \\
& +\frac{1}{2 !} f_{[N t] / N}^{\prime \prime}\left(t-\frac{[N t]}{N}\right)^{2}+\frac{1}{3 !} f_{[N t] / N}^{\prime \prime \prime}\left(t-\frac{[N t]}{N}\right)^{3}+\ldots
\end{aligned}
$$

on the ith time interval $i / N \leq t \leq(i+1) / N$. In Eq. (6), the number of terms to be remained is determined as per the desired order of accuracy.

With the form showing in Eq. (6), a complete solution is readily available. Most numerical simulations seeing in the field for solving nonlinear dynamic problems are with the accuracy of $4^{\text {th }}$ order, such as the Runge-Kutta method. In this research, the $4^{\text {th }}$ order accuracy is considered. As such, the solution for the governing equation Eq. (3) Eq. (6) may take the following form

$$
x_{i}(t)=B_{1}+B_{2} e^{-k\left(t-\frac{[N t]}{N}\right)}+A_{1}+A_{2}\left(t-\frac{[N t]}{N}\right)
$$

$$
+A_{3}\left(t-\frac{[N t]}{N}\right)^{2}+A_{4}\left(t-\frac{[N t]}{N}\right)^{3}
$$

in which, as per Dai [10], the coefficients can be given by

$$
\begin{gathered}
A_{4}=\frac{1}{24 k} f_{[N t] / N}^{\prime \prime} \\
A_{3}=\frac{1}{3 k}\left(\frac{1}{2} f^{\prime \prime}{ }_{[N t] / N}-12 A_{4}\right) \\
A_{2}=\frac{1}{2 k}\left(f^{\prime}{ }_{[N t] / N}-6 A_{3}\right) \\
A_{1}=\frac{1}{k}\left(f_{[N t] / N}-2 A_{2}\right) \\
B_{2}=\frac{1}{k}\left(A_{1}-v_{i}\right) \\
B_{1}=\left(d_{i}-C_{2}\right)
\end{gathered}
$$

where

$$
\begin{gathered}
f_{[N t] / N}=d_{i}^{3}-d_{i}^{5}+r \cos \omega t \\
\ddot{x}([N t] / N)=f-k v_{i} \\
f_{[N t] / N}^{\prime}=3 d_{i}^{2} v_{i}-5 d_{i}^{4} v_{i}-r \omega \sin \omega t
\end{gathered}
$$

$$
\begin{aligned}
& f_{[N t] / N}^{\prime \prime}=6 d_{i} v_{i}^{2}+3 d_{i}^{2}\left(f-k v_{i}\right)-\left(20 d_{i}^{3} v_{i}^{2}+5 d_{i}^{4}\left(f-k v_{i}\right)\right) \\
& -r \omega^{2} \cos \omega t
\end{aligned}
$$

$$
\begin{aligned}
& f_{[N t] / N}^{\prime \prime \prime}=6 v_{i}^{3}+18 d_{i} v_{i} \ddot{x}+3 d_{i}^{2}\left(f^{\prime}-k \ddot{x}\right) \\
& -\left(60 d_{i}^{2} v_{i}^{3}+60 d_{i}^{3} v_{i} \ddot{x}+5 d_{i}^{4}\left(f^{\prime}-k \ddot{x}\right)\right)+r \omega^{3} \sin \omega t
\end{aligned}
$$

For the solution such derived, the first derivative of the solutions in Eq. (7) is readily available, as per Dai [10]. It should be noticed that the Duffing system considered is continuous as it should be in reality. The conditions of continuity for the systems in the following form are assumed true over the time domain considered.

$$
x_{i}\left(\frac{i}{N}\right) \triangleq x_{i-1}\left(\frac{i}{N}\right), \quad \dot{x}_{i}\left(\frac{i}{N}\right) \triangleq \dot{x}_{i-1}\left(\frac{i}{N}\right)
$$

This is to say that the solutions such obtained by the P-T method are continuous everywhere, not only over the time interval $i / N \leq t \leq(i+1) / N$ but also the entire time range considered. The accuracy of the solutions depends on the value $N$ selected and the number of Taylor expansion terms used. Such solution is therefore semianalytical, yet, the solution can be exact when $N$ approaches infinity.

With the solutions obtained, the following recurrence relation for numerical calculations can be derived and the numerical calculations can thus be conducted, together with the conditions of continuity, system parameter and initial conditions for the system considered.

$$
x_{i+1}=B_{1}+B_{2} e^{-\frac{k}{N}}+A_{1}+\frac{A_{2}}{N}+\frac{A_{3}}{N^{2}}+\frac{A_{4}}{N^{3}}
$$




\section{Numerical results and discussions}

\subsection{Accuracy comparison between P-T and Runge-Kutta methods}

For numerical analysis of any system, accuracy of the numerical results calculated is always a consideration. The accuracy of the P-T method has been identified and approved theoretically and numerically by Dai and Wang [9]. Although Runge-Kutta method is probably the most popular method in numerically solving for nonlinear dynamic problems, P-T method has shown obvious advances for solving the same problems. In this section, the P-T method is to be compared with that of the Runge-Kutta method, in numerically solving for the Duffing systems governed by Eq. (1). The results generated by the two methods are shown in Figure 1a and 1b. As shown in Figure 1a, when the behavior of the system is regular, the results generated by both P-T and Runge-Kutta method are almost identical by naked eyes.

To emphasize the numerical differences of the results generated by the two methods, the following index is introduced.

$$
R(t)=\frac{\left|x_{P-T}-x_{R K}\right|}{\left|x_{P-T}\right|}
$$

The differences of the two methods are shown in Figure $1 \mathrm{~b}$, from which the difference between the two methods is indeed small enough to be ignorable.

For irregular behavior however, especially for chaotic responses, which are very sensitive the initial conditions and sensitive to computational errors as well, the comparisons of the numerical results obtained by the two methods are observable, as shown in Figure 2a and 2b, respectively. One may recognize, as theoretically proved by Dai and Wang $[9,10]$, the numerical results generated by the P-T method are more accurate and reliable than that of the Runge-Kutta method.

\subsection{Evaluation of nonlinear characteristics of the system with P-R method}

There are several methods in the field available for analyzing the characteristic patterns of nonlinear dynamic systems. Among the methods, the Lyapunov-Exponent is probably the most popular method used. However, the Lyapunov-Exponent method may not be applicable for predicting systems that are neither periodic nor chaotic. As an alternative, Periodicity-Ratio method provides a sin-

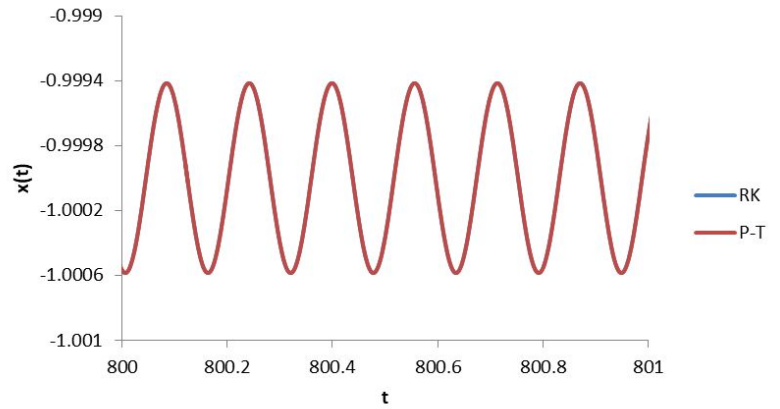

(a)

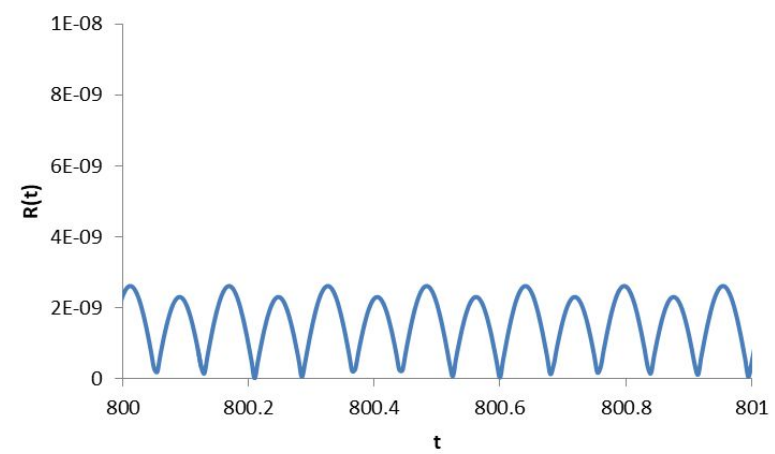

(b)

Figure 1: a) Numerical results of P-T and Runge-Kutta method ( $d t=$ $0.005, r=0.935, \omega=40, k=0.5, x(0)=-1.0, \dot{x}(0)=-1.0)$; b) Comparison of P-T and Runge-Kutta method ( $d t=0.005, r=0.935, \omega=40, k=0.5$, $x(0)=-1.0, \dot{x}(0)=-1.0)$

gle value index, the P-R value. With the P-R method, generated by Dai [10], the nonlinear behavior of a dynamic system can be conveniently studied. Significantly, the nonlinear behavior what is neither chaotic nor periodic can be quantitatively described. Moreover, a diagram graphically demonstrates the nonlinear behaviors of a dynamic system with respect to varying system parameters can be constructed with the single value index..

As per the P-R method, the Periodicity Ratio or P-R value can be defined as:

$$
y=\lim _{n \rightarrow \infty} \frac{N O P}{n}
$$

where NOP in the definition represents the total number of points periodically appearing in a specified Poincare map. In Eq. (11), $n$ is the total points determined for constructing the Poincare map.

Mathematically, the NOP can be given as:

$$
N O P=\zeta(1)+\sum_{k=2}^{n} \zeta(k) \cdot P\left(\prod_{l=1}^{k-1}\left\{X_{k l}+\dot{X}_{k l}\right\}\right)
$$

in which $\prod$ is the symbol for multiplication and $\zeta(k)$ represents the total number of points overlapping with the $k$ th 


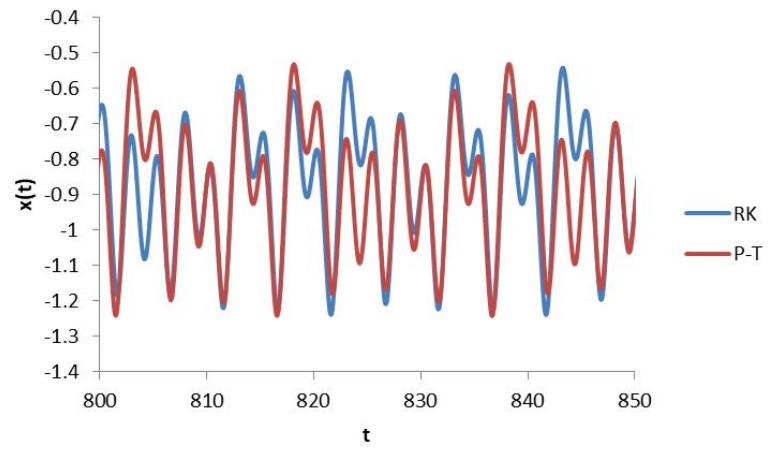

(a)

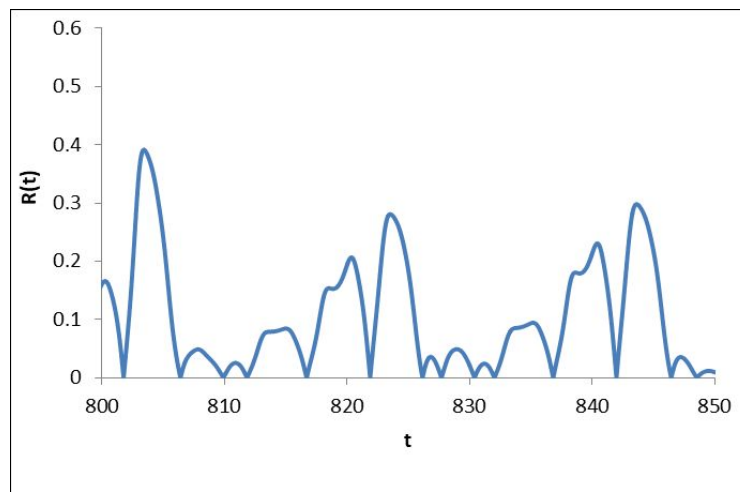

(b)

Figure 2: a) Numerical results of P-T and Runge-Kutta method ( $d t=$ $0.005, r=0.935, \omega=2.5, k=0.5, x(0)=-1.0, \dot{x}(0)=-1.0)$; b) Numerical comparison of P-T and Runge-Kutta method $(=0.005, r=0.935$, $\omega=2.5, k=0.5, x(0)=-1.0, \dot{x}(0)=-1.0)$

point in the Poincare map. The $X_{k l}, \dot{X}_{k l}$ are functions that determine whether the $k$ th and $i$ th points are overlapped with each other

$$
\begin{aligned}
& X_{k l}=\left|X\left(\tau_{0}+k T\right)-X\left(\tau_{0}+l T\right)\right| \\
& \dot{X}_{k l}=\left|\dot{X}\left(\tau_{0}+k T\right)-\dot{X}\left(\tau_{0}+l T\right)\right|
\end{aligned}
$$

The function $P$ is step function represented as

$$
P(z)=\left\{\begin{array}{lll}
0 & \text { if } & z=0 \\
1 & \text { if } & z \neq 0
\end{array}\right.
$$

In employing the Periodicity Ratio (P-R) as an index, the following conclusions can be given.

- When $y$ equals one, all the points in the Poincare map are periodically appearing, and the response of the system is periodic or as known as regular.

- When P-R value $y$ is zero, no point in the Poincare map is overlapping with the other points. The response of the system is either chaotic or quasiperiodic, or as known as irregular.
- When the P-R values fall in the between 0 and 1, $0<$ $y<1$ the corresponding responses of the system are nonperiodic or combinations of periodic and nonperiodic motions.

- For the sake of clarity, in this research, the period cases are considered as regular and all the other cases are designated as irregular.

For identifying and analyzing the nonlinear behaviors of the Duffing equation considered, constructing a regular and irregular diagram (region diagram) is significant. The diagram can be used to effectively identify the nonlinear behaviors and consider the interested system parameters over desired ranges. Such a diagram is practically sound with variation of usually two system parameters considered. Difference shapes and colors in the diagram represent different PR values. Therefore, the distribution of the PR values with corresponding system parameters and initial conditions can be captured in this diagram. Since each PR value can represent a state of motion, such as periodic motions or nonperiodic motions, the state of motion of the system under certain system parameters can be easily predicted using the region diagrams.

For the system shown in Eq. (1), a region diagram with $y$ and $\omega$ selected as the system parameters is constructed and plotted in Figure 3, by employing the P-R index. For ensuring the high accuracy and reliability of the numerical solutions, all the solutions for the system are determined by utilizing the P-T method.

In using the P-T method for the calculations, the time step used is 0.001s. In Figure 3, the diagram consists of $35640(180 \times 198)$ points and each point actually represents a state of the nonlinear system, as per its P-R value. The green squares in the diagram demonstrate irregular points (states) where $y=0$, and the red squares in the diagram represents regular regions of which $y=1$. The blank regions without any marks denote the responses of the system neither regular nor irregular, specifically, $0<y<1$. The region diagram provides an overall view of the characteristics of the nonlinear dynamic system investigated.

It can be seen from the region diagram plotted; most of the patterns in the nonlinear system described by Eq. (1) perform regularly with stable state corresponding to large ranges of parameters. The examined amplitude $\omega$ plays an insignificant role in changing characteristics of the system. When the external angular velocity is below around 10 , the overall nonlinear dynamic system appears irregular, nonperiodic and unstable.

It should be stated that more system parameters can be taken into considerations if so desire, though only two 


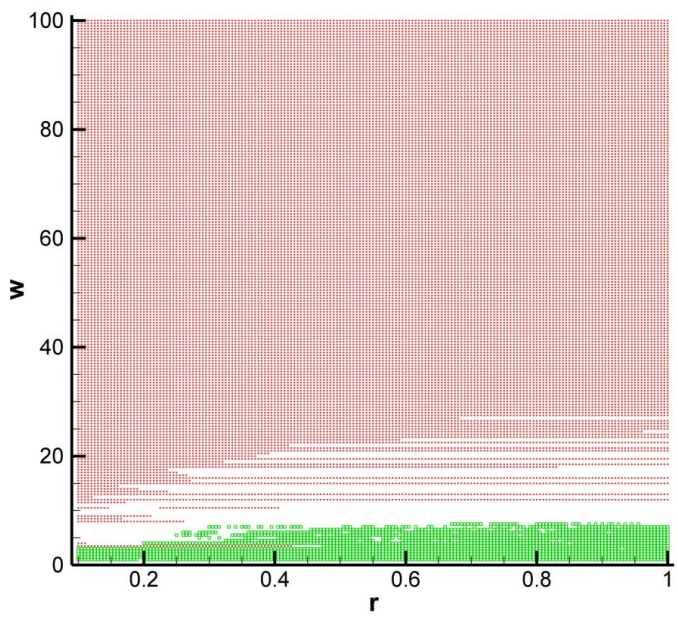

Figure 3: Region diagram with varying system parameters $r$ and $\omega$ $(k=0.5)$

system parameters, the amplitude $r$ and the angular velocity $\omega$, are considered for the diagram shown in Figure 3.

Figure 4 demonstrates the non-linear dynamic of regular motion with the P-R value equaling to one. The curve wave or a time history of the motion exhibits a visible pattern showing a perfect periodic motivation as shown in Figure 4a, and its responding phase trajectory, presented in Figure $4 \mathrm{~b}$ forms a closed curve. What is more, only one point can be seen in the Poincare map, in Figure 4c, which implies the corresponding pattern, is actually a simple harmonic response.

A more complex periodic case is taken and shown in Figure 5. The parameters of this kind of response are similar to those of irregular motion because of the visible points distributing in transitional zone of the region diagram. It can be seen from the wave curve Figure 5a, corresponding phase diagram Figure 5b, and Poincare map Figure $5 \mathrm{c}$ with more than one overlapping point, even though the movement repeats during a certain amount of time, the general track is more complex than that shown in Figure 4.

A specific case for irregular behavior of the system is shown in Figure 6. It demonstrates an example with distribution located in the irregular motion region, where the external excitation and the angular velocity are assigned fairly low values. The corresponding amplitude and frequency in its wave curve in Figure $6 \mathrm{a}$ are irregular and the phase diagram in Figure $6 \mathrm{~b}$ also forms a shape of irregularity. In terms of the Poincare map in Figure 6c, the points are randomly scattered, which is a typical chaotic case.

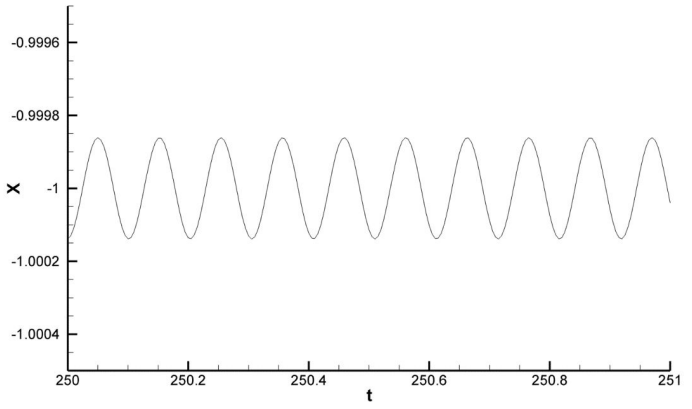

(a) Wave curve

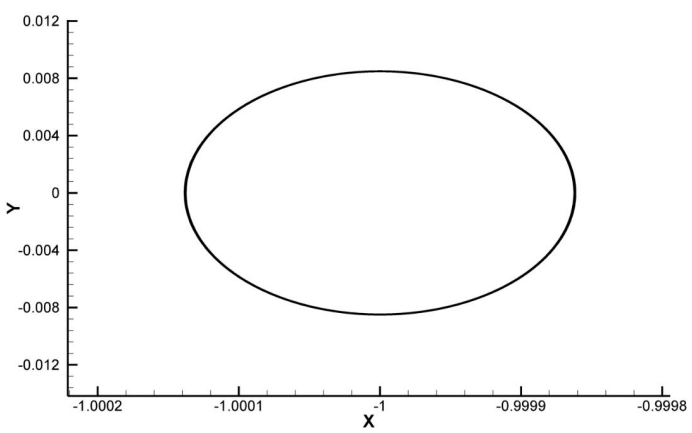

(b) Phase diagram

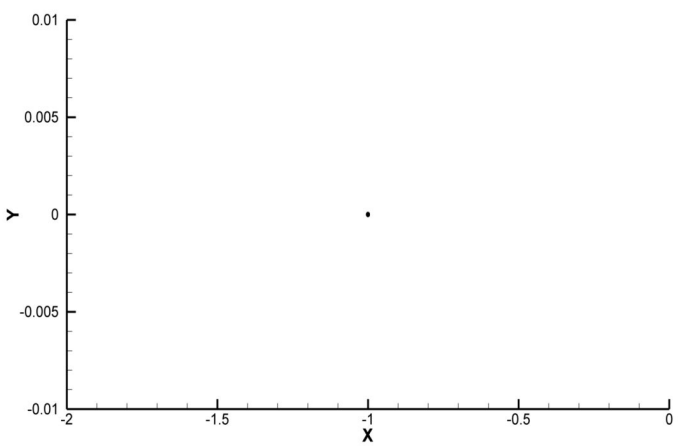

(c) Poincare map

Figure 4: Periodic behavior of non-linear system $(r=0.523, \omega=$ 61.5, $k=0.5, x(0)=-1.0, \dot{x}(0)=-1.0)$

The chaotic response is complex and difficult to predict for a long run since it is much more sensitive to the initial conditions and computing accuracy. When solving such systems, method with high accuracy and reliability is a must to be considered. This gives the rational for utilizing the P-T method for the numerical calculations. 


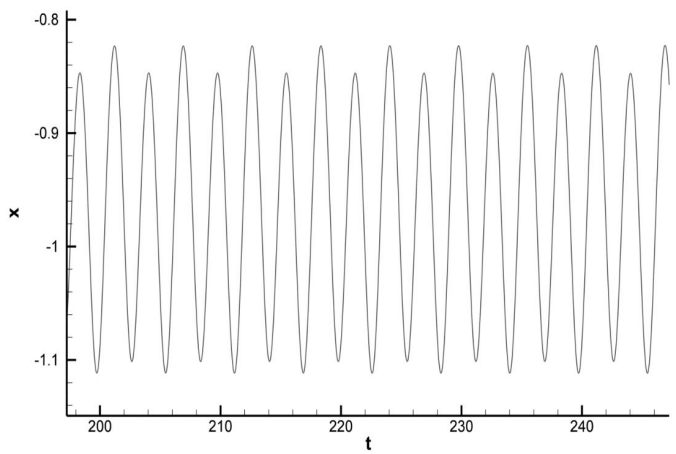

(a) Wave curve

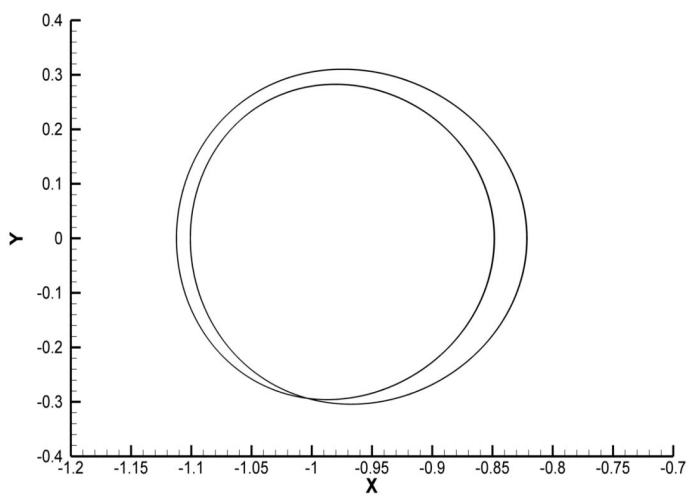

(b) Phase diagram

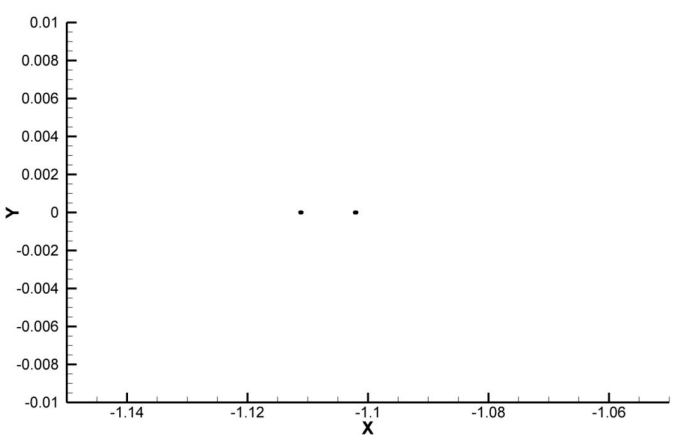

(c) Poincare map

Figure 5: Complex periodic behavior of non-linear system $(r=0.45$, $\omega=2.2, k=0.5, x(0)=-1.0, \dot{x}(0)=-1.0)$

\subsection{Investigation of the reliability of P-T method}

For demonstrate the intrinsic characteristics of the P-T method over that of the Runge-Kutta method, the region diagrams of the Duffing system shown in Eq. (1) are con-

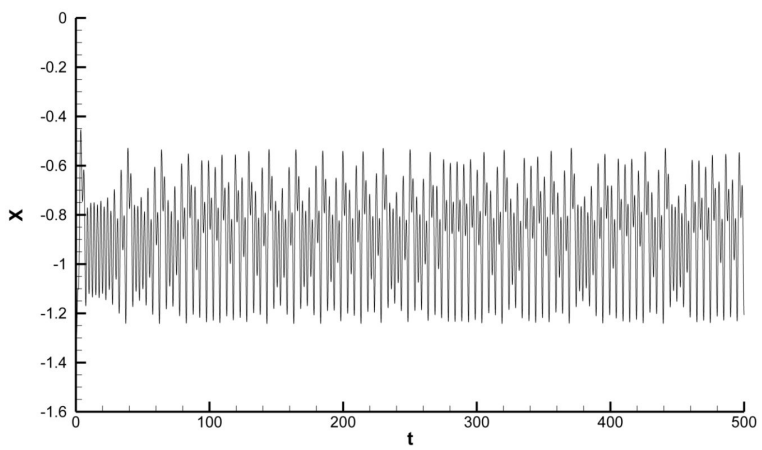

(a) Wave curve

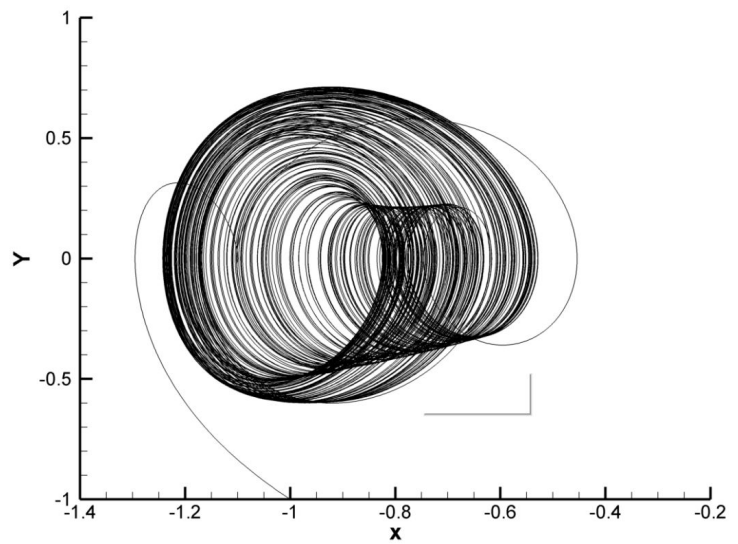

(b) Phase diagram

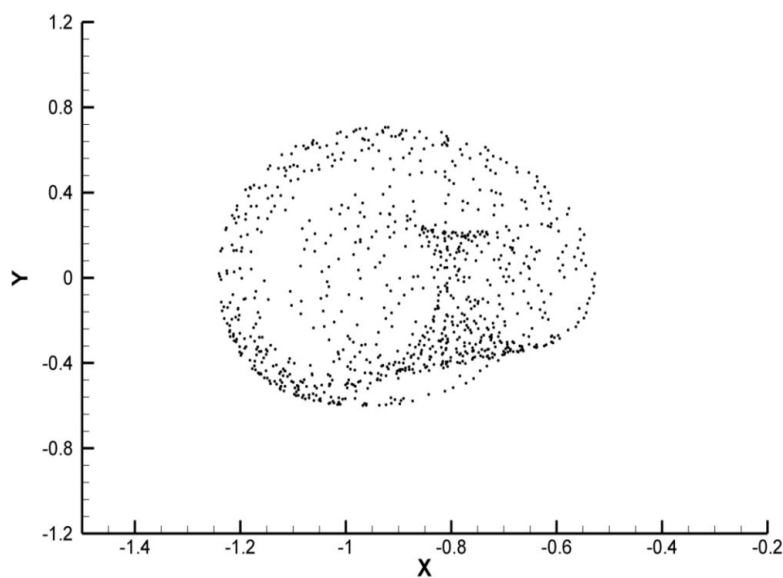

(c) Poincare map

Figure 6: Chaotic Behavior of non-linear system $(r=0.935, \omega=2.5$, $k=0.5, x(0)=-1.0, \dot{x}(0)=-1.0)$

structed with the two methods respectively, with exactly the identical system parameters and under the same numerical calculation conditions.

It can be seen that the two figures, Figure $7 \mathrm{a}$ and $7 \mathrm{~b}$ are almost identical at most of areas in the diagrams. However, there are still quite a few naughty points in the diagrams 


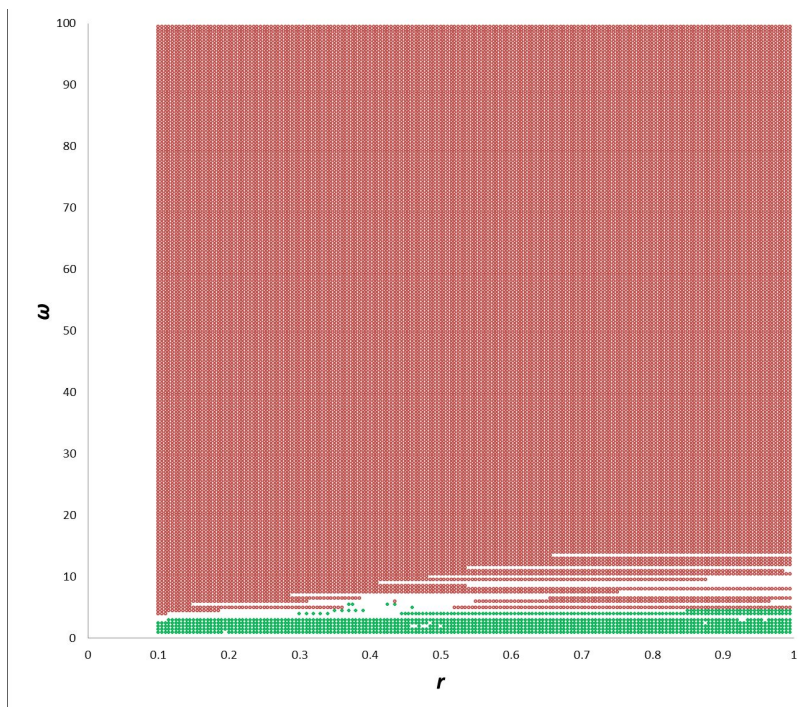

(a) Region diagram calculated by P-T method

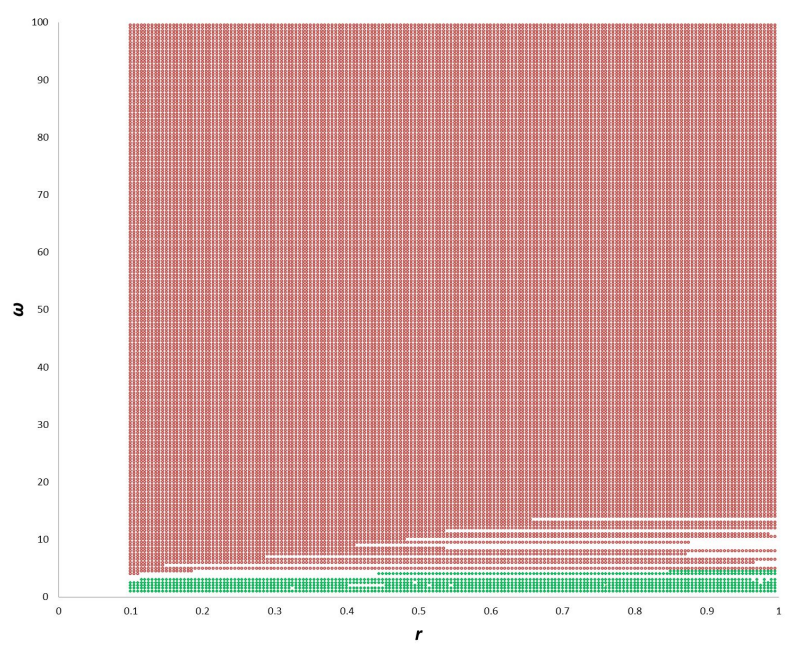

(b) Region diagram calculated by Runge-Kutta method

Figure 7: Region diagrams generated by P-T and Runge-Kutta methods $(x(0)=-1.0, \dot{x}(0)=-1.0)$

different from each other. Take point $\omega=5.0, r=0.46$ for example, the comparison of the results calculated by these two methods are presented in the Figure 8. Even when we further reduce the time step, the RK method is still generating the same regular motion as presented in Figure 9. The results under the two time steps are overlapping with each and the difference between is un-recognizable.

It can be seen that the P-T method demonstrates an irregular case while the Runge-Kutta method shows a different idea. Even if we future reduce the time step used in the calculation, the Runge-Kutta method still shows the same regular motion. This leads to the implication that Runge-Kutta method is less accurate than that of the P-T method and may even lead to incorrect conclusions. This

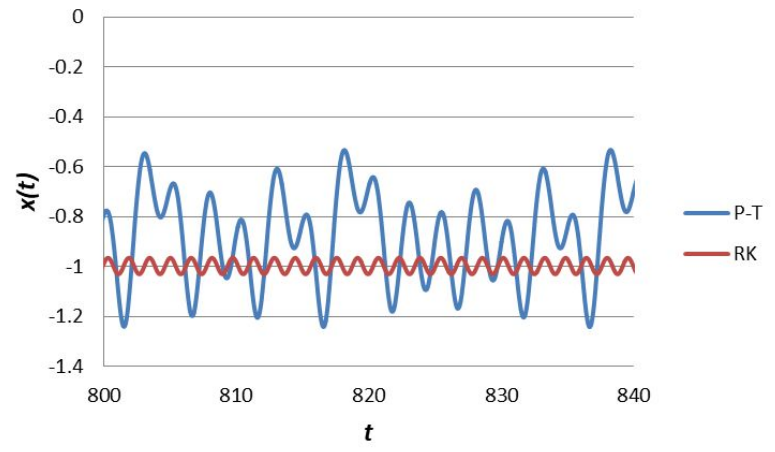

Figure 8: Comparison of wave diagrams $(d t=0.001, x(0)=-1.0$, $\dot{x}(0)=-1.0)$

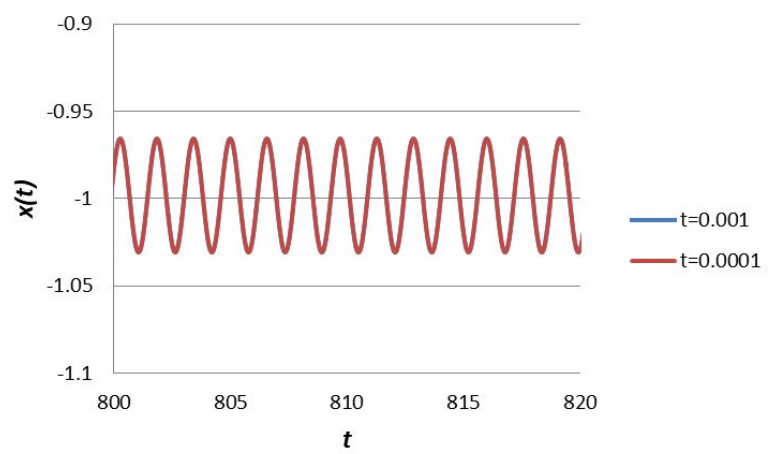

Figure 9: Wave diagram under different time step for RK method ( $d t$ $=-1.0, \dot{x}(0)=-1.0$ )

is due to the damage caused by the Runge-Kutta method to the physical information in the original system. The PT method, however, tries to maintain as much as possible the original physical information in the system unchanged. The results of the P-T method are therefore more accurate and reliable. It may worth to mention here that the solution created by the P-T method, shown in Eq. (7), is actually continuous over the entire time range considered, in comparing with the discrete solutions derived by the Runge-Kutta method. Furthermore, the continuous solution of the P-T method can be the exact solution as $N$ in Eq. (7) approached infinity.

Nonlinear especially chaotic behavior of a dynamic system seeing in industry is usually harmful and need to be eliminated or avoided. With the region diagrams provided, the state of motion of the systems corresponding to the desired system parameters and the initial conditions considered can be predicted easily and quantitatively. This would provide a guidance to the practical design and manufacturing. Base on the region diagrams, the design and operation of a dynamic system can be such conducted to achieve preferred motions of the system with desired char- 
acteristics while being avoided for unwanted behaviors. It should be noticed that there is no restrictions on the range selections of the system parameters and three or more system parameters can be considered with the approach presented, if in any case needed.

\section{Conclusion}

This research investigates the behavior of a nonlinear Duffing's system applied in traffic flow prediction. It is suggested that the P-T method should be employed for assuring high efficiency in performing the traffic flow prediction with identification of the nonlinear patterns of the Duffing system considered. By the results of the research, the P-T method produces more accurate and reliable solutions in comparison with the Runge-Kutta method; which is the numerical method probably has the highest popularity in the field of nonlinear dynamics. Because that the crucial part of the nonlinear dynamic system plays an important role in traffic flow prediction investigation, the application of the P-T method is expected to contribute to more accurate and reliable traffic pattern recognition. Furthermore, regular-irregular diagrams with respect to the system parameters show great effectiveness for identifying and analyzing the nonlinear patterns especially the irregular patterns of the system. The P-R index is evidently an effective tool for generating such practically sound diagrams in the research. It is also found in the research, when the frequency of the external excitation is properly low, the system behavior can be either chaotic or quasiperiodic. The system can be stabilized and reduced to periodic when the frequency is large enough.

Conflict of interest: The authors declare no conflict of interest regarding the publication of this paper.

\section{References}

[1] Zhang N., Wang F. Y., Zhu F., Zhao D., Tang, S., Dyna CAS: Computational experiments and decision support for ITS, IEEE Intell. Syst., 2008, 23(6), 19-23.

[2] Lv Y., Duan Y., Kang W., Li Z., Wang F., Traffic Flow Prediction with Big Data: A Deep Learning Approach, IEEE Trans. Intell. Transport. Syst., 2014, 16(2), 865-873.

[3] Mrgole A. L., Drago, S., Incorporation of Duffing Oscillator and Wigner-Ville Distribution in Traffic Flow Prediction, PROMETTraffic \& Transport., 2017, 29(1), 13-22.

[4] Yang Z., Bing Q., Lin C., Yang N, Duo M., Research on ShortTerm Traffic Flow Prediction Method Based on Similarity Search of Time Series, Math. Probl. Eng., 2014(1), 1-8.

[5] Shampine L.F., Watts, H. A., Comparing error estimators for Runge-Kutta methods, Math. Comput., 1971,25(115), 445-455.

[6] Hull T. E., Enright B.M., Fellen B. M., Sedgwick A.E., Comparing numerical methods for ordinary differential equations, SIAM J. Numer. An., 1972, 9(4), 603-637.

[7] Li K., Darby, A., A high precision direct integration scheme for nonlinear dynamic systems, ASME J. Comput. Nonlin. Dyn., 2009,4(4), 041008.

[8] Enright W.H., Hayes W.B., Robust and reliable defect control for Runge-Kutta methods, ACM Trans. Math. Softw., 2007, 33(1): 1-19.

[9] Dai L., Wang X., Accuracy and Reliability of P-T Method in Studying the Responses of Nonlinear Dynamic Systems, ASME J. Comput. Nonlin. Dyn., 2014, 10(2), 021009.

[10] Dai L., Nonlinear Dynamics of Piecewise Constant Systems and Implementation of Piecewise Constant Arguments, World Scientific Publishing Co., New Jersey, 2008.

[11] Dai L., Zhang Y., Wang X., Huang, T., Nonlinear Oscillations of an Articulated Pipe System Subjected to Oil Flow and External Excitations, J. Appl. Nonlin. Dyn., 2014, 3, 255-269.

[12] Huang T., Dai L., Zhang H., An Approach combining periodicity ratio and secondary Poincare Map for Characteristics Diagnosis of Nonlinear Oscillatory Systems, Nonlin. Dyn., 2015, 84(2), 959-975.

[13] Dai L., Xia D., Chen C., An Algorithm for diagnosing Nonlinear Characteristics of Dynamic Systems with the Integrated Periodicity Ratio and Lyapunov Exponent Methods, Comm. Nonlin. Sci. Numer. Simul., 2019, 73, 92-109.

[14] Kovacic I., Brennan M., The Duffing Equation: Nonlinear Oscillators and their Behaviour, John Wiley \& Sons, West Sussex, 2011.

[15] Dai L., Singh M.C., Diagnosis of periodic and chaotic responses in vibratory systems, J. Acoust. Soc. Amer., 1997, 102(6), 3361- 3371. 